\title{
Toward an effective theory of quarkonium production in nuclear matter
}

\author{
Ivan Vitev* \\ Los Alamos National Laboratory, Theoretical Division, Mail Stop B283, Los Alamos, NM 87544 \\ E-mail: ivitev@lanl.gov
}

\begin{abstract}
These proceedings are dedicated to Miklos Gyulassy's $70^{\text {th }}$ birthday. In his long and distinguished career he has made seminal contributions to many areas of heavy ion theory, including early papers on quarkonium phenomenology in fixed-target heavy ion experiments. Theoretical and experimental studies of the $J / \psi$ and $\Upsilon$ states have evolved considerably in the past decades, and I describe a recent generalization of non-relativistic Quantum Chromodynamics to include interactions in a generic nuclear medium. NRQCD with Glauber gluons aims to provide a universal microscopic description of the interaction of heavy quarkonia in a range of phases that include cold nuclear matter, dense hadron gas, and quark-gluon plasma. Such effective field theory is an important step toward understanding the common trends in proton-nucleus and nucleus-nucleus data on quarkonium suppression.
\end{abstract}

13th International Workshop in High pT Physics in the RHIC and LHC Era (High-pT2019)

19-22 March 2019

Knoxville, Tennessee, USA

\footnotetext{
*Speaker.
} 


\section{Introduction}

A significant part of Miklos Gyulassy's research has been dedicated to the physics of jet quenching, as described in other talks in this symposium [1]. In the late 1980s, together with Gavin and Jackson, he co-authored a couple of paper on $J / \psi$ modification in fixed-target $\mathrm{O}+\mathrm{U}$ collisions [2, 3], pointing out that quarkonium suppression can occur not only in the QGP but also in a hadron gas. This observation remains pertinent today, as experimental measurements of the suppression of excited versus ground bottomonium states $\Upsilon(2 S) / \Upsilon(1 S)$ as a function of the number of charged particle tracks, shows the same trend for high-multiplicity $\mathrm{p}+\mathrm{p}, \mathrm{p}+\mathrm{Pb}$, and $\mathrm{Pb}+\mathrm{Pb}$ collisions at the LHC [4]. $\psi^{\prime}, \chi_{c}$ and $\Upsilon$ suppression in d+Au reactions was also measured at RHIC [5, 6].

To address the modification of quarkonium cross sections in $\mathrm{p}+\mathrm{A}$ and $\mathrm{A}+\mathrm{A}$ reactions, we aim to develop a universal microscopic theory of $J / \psi \mathrm{s}$ and $\Upsilon \mathrm{s}$ applicable to different phases of nuclear matter. The effective field theory approach is particularly suitable, as it can provide a modelindependent description of the universal physics of energetic particle production in the background of a QCD medium. It has been applied to light and open heavy flavor final states to formulate a new theory - soft collinear effective theory with Glauber gluons $\left(\operatorname{SCET}_{\mathrm{G}}\right)[7,8]$.

With this in mind, we first observe that calculations of heavy quarkonium production involve hierarchies of momentum and mass scales. These scales are $p_{T}, m_{Q}, m_{Q} \lambda, m_{Q} \lambda^{2}$, and $\Lambda_{\mathrm{QCD}}$, where $p_{T}$ is the quarkonium transverse momentum, $m_{Q}$ the heavy quark mass, and $\lambda$ the heavy quark-antiquark pair relative velocity. The established and most successful effective theory that describes quarkonium production and decays is non-relativistic QCD (NRQCD) [9]. As in the vacuum, production of quarkonia in nuclear matter remains a multi-scale problem. We have recently demonstrated that one can generalize NRQCD to incorporate interactions of the non-relativistic heavy quarks with the medium [10]. This was achieved by incorporating the Glauber and Coulomb gluon exchanges of the heavy quarks with the quasiparticles of QCD matter. We believe this version of NRQCD will facilitate a much more robust and accurate theoretical analysis of the wealth of existing and upcoming quarkonium measurements.

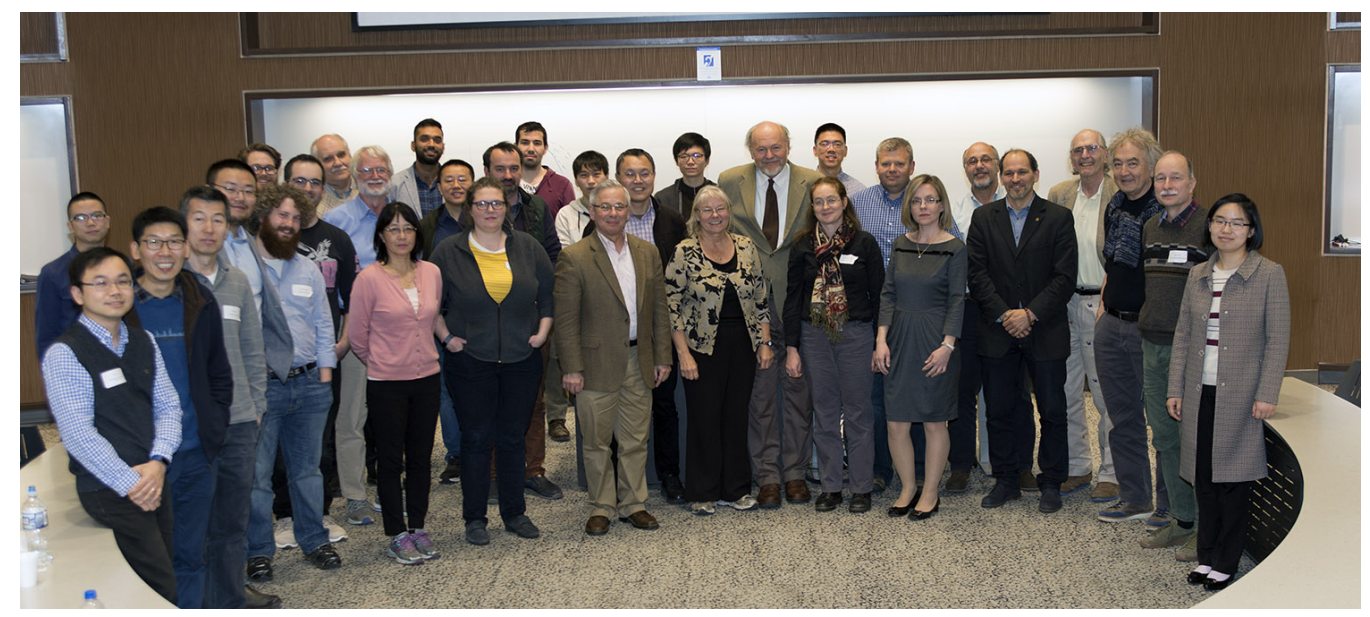

Figure 1: Miklos Gyulassy and Gyorgyi Gyulassy with colleagues and friends at the $13^{\text {th }}$ International Workshop in High $p_{T}$ Physics in the RHIC and LHC Era. 
In Section II I will first show that the successful jet quenching approach is challenged by the totality of the ground and excited charmonium state measurements. In Section III the formulation of $\mathrm{NRQCD}_{\mathrm{G}}$ is described. The Lagrangian of the new theory to leading and subleading power is written down in Section IV. I conclude these proceedings in Section V.

\section{Energy loss approach to quarkonium production}

Jet quenching $[11,12]$ is the physical mechanism behind the suppression of high transverse momentum particles and jets in ultrarelativistic nuclear collisions. Understandably, it has been suggested $[13,14]$ that energy loss effects can reduce the cross section of $J / \psi$ production at the LHC $[15,16]$. In Ref. [10] we revisited this conjecture making use of the leading power (LP) factorization of NRQCD $[17,18]$, which is expected to hold at high transverse momenta $\left(p_{T} \gg m_{Q}\right)$. In this limit the production cross section is factorized into short distance matching coefficients that describe the production and propagation of a parton $k$ and the NRQCD fragmentation functions,

$$
d \sigma_{i j \rightarrow \mathscr{Q}+X}\left(p_{T}\right)=\sum_{n} \int_{x_{\min }}^{1} \frac{d x}{x} d \sigma_{i j \rightarrow k+X^{\prime}}\left(\frac{p_{T}}{x}, \mu\right) D_{k / \mathscr{Q}}^{n}(x, \mu) .
$$

The dependence on the factorization scale, $\mu$, allows for the resummation of large logarithms through the use of renormalization group techniques such as the DGLAP evolution of the fragmentation functions. The NRQCD fragmentation functions can be written in terms of the same long-distance matrix elements (LDMEs) that appear in the fixed order formulas,

$$
D_{k / \mathscr{Q}}^{n}(x, \mu)=\frac{\left\langle\mathscr{O}^{\mathscr{Q}}(n)\right\rangle}{m_{c}^{[n]}} d_{k / n}(x, \mu),
$$

where $[n]=0$ for $\mathrm{S}$-wave and $[n]=2$ for P-wave quarkonia. The short distance coefficients, $d_{k / n}(x, \mu)$, are functions of the fraction, $x$, of the parton energy transferred to the quarkonium state. Recent phenomenological applications to charmonia show that Eq. (2.1) may hold to transverse momenta $p_{T} \sim 10 \mathrm{GeV}$ [19]. In this work we also considered the $J / \psi$ feed-down from decays of excited quarkonium states $-\psi(2 S): \operatorname{Br}[\psi(2 S) \rightarrow J / \psi+X]=61.4 \pm 0.6 \%, \chi_{c 1}: \operatorname{Br}\left[\chi_{c 1} \rightarrow\right.$ $J / \psi+\gamma]=34.3 \pm 1.0 \%, \chi_{c 2}: \operatorname{Br}\left[\chi_{c 2} \rightarrow J / \psi+\gamma\right]=19.0 \pm 0.5 \%$

Within the energy loss approach the cross section for and quarkonium production per elementary nucleon-nucleon collision in the leading power limit can then be expressed as

$$
\frac{1}{\left\langle N_{\text {coll. }}\right\rangle} \frac{d \sigma_{m e d}^{h}}{d y d^{2} p_{T}}=\sum_{c} \int_{z_{\min }}^{1} d z \int_{0}^{1} d \varepsilon P(\varepsilon) \frac{d \sigma^{c}\left(\frac{p_{T}}{(1-\varepsilon) z}\right)}{d y d^{2} p_{T_{c}}} \frac{1}{(1-\varepsilon)^{2} z^{2}} D_{h / c}(z) .
$$

Here, $P(\varepsilon)$ is the probability distribution for the hard parton $c$ to lose energy due to multiple gluon emission, $\frac{d \sigma^{c}\left(p_{T}\right)}{d y d^{2} p_{c}}$ is the hard partonic cross section, and $\left\langle N_{\text {coll. }}\right\rangle$ is the average number of binary nucleon-nucleon collisions. We use the soft gluon emission limit of the full medium induced splitting function, which have been recently applied to light and heavy flavor jets and jet substructure $[20,21,22]$. Comparison our theoretical calculations to ATLAS data on the transverse momentum dependence of $J / \Psi$ attenuation from $0-10 \%$ central $\sqrt{s_{N N}}=5.02 \mathrm{TeV} \mathrm{Pb}+\mathrm{Pb}$ collisions at the LHC [16] is shown in the left panel of Figure 2. The data is not described by the 

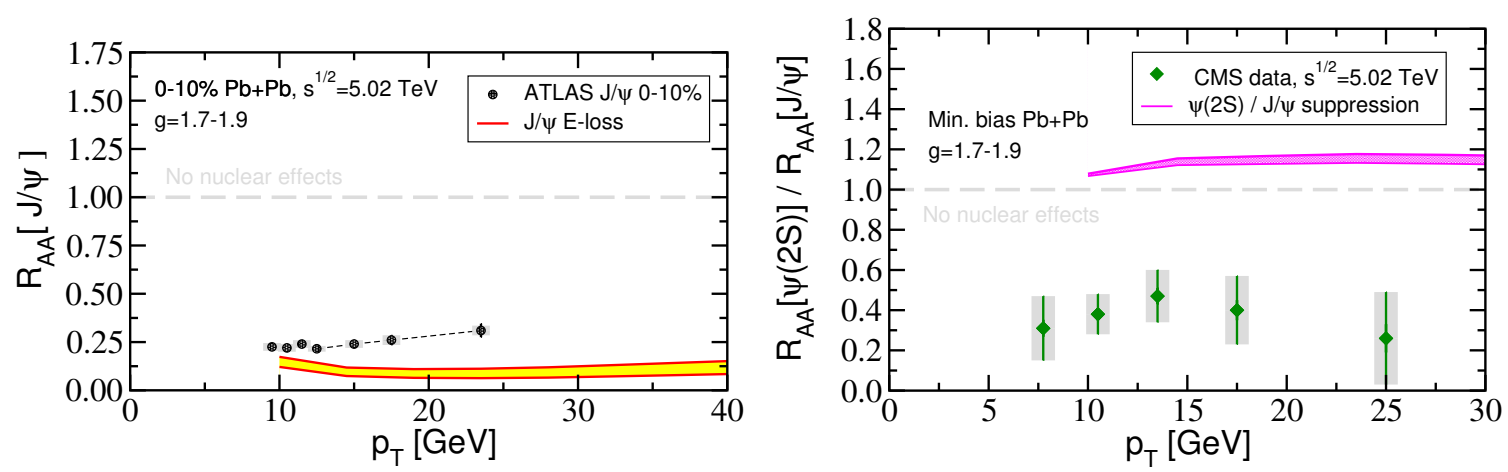

Figure 2: Left: comparison of the suppression of $J / \psi$ (yellow band) evaluated in an energy loss model with coupling between the parton and the medium $g=1.7-1.9$ to ATLAS data from $\sqrt{s_{N N}}=5.02 \mathrm{TeV} \mathrm{Pb}+\mathrm{Pb}$ collisions at the LHC [16]. Right: the double ratio of $\psi(2 S)$ to $J / \psi$ suppression [15] as a measure of the relative significance of QCD matter effects on ground and excited states is compared to energy loss model calculations (purple bands). Figures are reproduced from Ref. [10].

theoretical predictions. Energy loss calculations overpredict the suppression of $J / \psi$ and at high $p_{T}$ the discrepancy is as large as a factor of 3. Even more importantly, in the right panel of Figure 2 we show the relative medium-induced suppression of $\psi(2 S)$ to $J / \psi$ in matter. The energy loss model yields a slightly smaller suppression for the $\psi(2 S)$ state when compared to $J / \psi$. Conversely, the experimental results show that the suppression of the weakly bound $\psi(2 S)$ is 2 to 3 times larger than that of $J / \psi$. It is clear that the energy loss model is incompatible with the hierarchy of excited to ground state suppression of quarkonia in matter.

\section{Non-relativistic QCD with Glauber gluons}

In formulating a generic framework of quarkonium propagation in a variety of strongly-interacting media we are interested in the regime where matter itself might be non-perturbative, but the interaction with its quasiparticles can be described by perturbation theory. When an energetic particle traverses QCD matter, the interaction with the scattering centers of the medium is typically mediated by $t$-channel exchanges of off-shell gluons, called Glauber gluons. We will call the new effective theory NRQCD with Glauber gluons, or NRQCD ${ }_{G}$. The Lagrangian of $\mathrm{NRQCD}_{\mathrm{G}}$ can be constructed by adding to the vNRQCD Lagrangian [23] the additional terms that include the interactions with quark and gluon sources through (virtual) Glauber/Coulomb gluons exchanges

$$
\mathscr{L}_{\mathrm{NRQCD}_{\mathrm{G}}}=\mathscr{L}_{\mathrm{vNRQCD}}+\mathscr{L}_{Q-G / C}\left(\psi, A_{G / C}^{\mu, a}\right)+\mathscr{L}_{\bar{Q}-G / C}\left(\chi, A_{G / C}^{\mu, a}\right) .
$$

In Eq. (3.1) the effective fields $A_{G / C}^{\mu, a}$ incorporate the information about the source fields, which can be collinear, static, or soft. To obtain the form and perform the power-counting of the terms in $\mathscr{L}_{Q-G / C}\left(\psi, A_{G / C}^{\mu, a}\right)$ we use three different approaches:

- The background field approach where we perform a shift in the gluon field in the NRQCD Lagrangian $\left(A_{u s}^{\mu} \rightarrow A_{u s}^{\mu}+A_{G / C}^{\mu}\right)$ and then perform the power-counting established in Table 1 to keep the leading contributions. 
- A hybrid method, where from the full QCD diagrams for single effective Glauber/Coulomb gluon insertion with appropriate power-counting one can extract the Feynman rules.

- A matching method where we expand in the power-counting parameter, $\lambda$, the full QCD diagrams describing the interactions of an incoming heavy quark and a light quark or a gluon.

Although there are subtleties involved in the background field method, the fact that all three approaches then give the same Lagrangian is a non-trivial test of our derivation.

For any gluon interacting with the vNRQCD heavy quark the scaling $q_{G / C}^{0} \sim \lambda^{2}$ and $q_{G / C}^{i} \lesssim \lambda$ is required so that the heavy quark momenta scale as $\left(\lambda^{2}, \lambda\right)$, see Figure 3 . If all of the threemomenta components scale as $\lambda$, i.e. $q_{C}^{\mu} \sim\left(\lambda^{2}, \lambda\right)$ then this corresponds to Coulomb (or potential) gluons. Collinear particles cannot interact with the heavy quarks through the exchange of Coulomb gluons since this will push the collinear particles away from their canonical angular scaling. The relevant mode here is the Glauber gluons, which scale as $q_{G}^{\mu} \sim\left(\lambda^{2}, \lambda, \lambda, \lambda^{2}\right)$. In this EFT we include Coulomb gluons for the interaction of the heavy quarks with soft and static modes and Glauber gluons for the interactions with collinear modes:

static and soft sources: $q_{C}^{\mu} \sim\left(\lambda^{2}, \lambda^{1}, \lambda^{1}, \lambda^{1}\right)$, collinear sources: $q_{G}^{\mu} \sim\left(\lambda^{2}, \lambda^{1}, \lambda^{1}, \lambda^{2}\right)$.

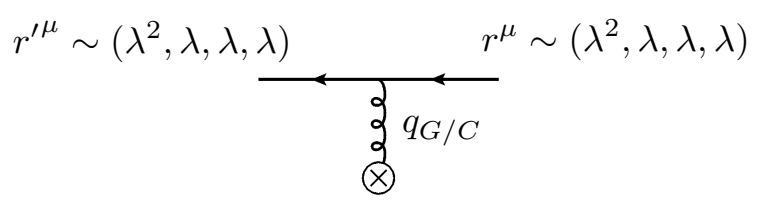

Figure 3: Single Glauber/Coulomb gluon insertion vertex from the Lagrangian $\mathscr{L}_{Q-G / C}$, where the incoming quark caries momentum $p^{\mu}=m v^{\mu}+r^{\mu}$ and the outgoing one carries momentum $p^{\prime \mu}=m v^{\mu}+r^{\prime \mu}$.

The scalings of the Glauber and Coulomb fields can be established for different sources of scattering in the medium and are presented in Table 1. Note that the scalings corresponds to the maximum allowed components for each source.

\begin{tabular}{|c|c|c|c|}
\hline Source & Collinear & Static & Soft \\
\hline \hline$A_{C}^{\mu} \sim$ & n.a. & $\left(\lambda^{1}, \lambda^{2}, \lambda^{2}, \lambda^{2}\right)$ & $\left(\lambda^{1}, \lambda^{1}, \lambda^{1}, \lambda^{1}\right)$ \\
\hline$A_{G}^{\mu} \sim$ & $\left(\lambda^{2}, \lambda^{3}, \lambda^{3}, \lambda^{2}\right)$ & n.a. & n.a. \\
\hline
\end{tabular}

Table 1: The Glauber/Coulomb filed scaling for different sources of interaction in matter as calculated in Ref. [10].

\section{The Lagrangian of $\mathrm{NRQCD}_{\mathrm{G}}$}

The details of the derivation of the $\mathrm{NRQCD}_{\mathrm{G}}$ Lagrangian using the three different methods discussed above can be found in our paper [10]. We summarize the leading and subleading terms 
in the Lagrangian that arise from the heavy quark sector coupling to the medium, i.e. $\mathscr{L}_{Q-G}$, from virtual gluon insertions:

$$
\mathscr{L}_{Q-G / C}^{(0)}\left(\psi, A_{G / C}^{\mu, a}\right)=\sum_{\mathrm{p}, \mathrm{q}_{T}} \psi_{\mathrm{p}+\mathrm{q}_{T}}^{\dagger}\left(-g A_{G / C}^{0}\right) \psi_{\mathrm{p}}(\text { collinear } / \text { static } / \text { soft }),
$$

and

$$
\begin{aligned}
& \mathscr{L}_{Q-G}^{(1)}\left(\psi, A_{G}^{\mu, a}\right)=g \sum_{\mathrm{p}, \mathrm{q}_{T}} \psi_{\mathrm{p}+\mathrm{q}_{T}}^{\dagger}\left(\frac{2 A_{G}^{\mathrm{n}}(\mathrm{n} \cdot \mathscr{P})-i\left[\left(\mathscr{P}_{\perp} \times \mathrm{n}\right) A_{G}^{\mathrm{n}}\right] \cdot \sigma}{2 m}\right) \psi_{\mathrm{p}}(\text { collinear }), \\
& \mathscr{L}_{Q-C}^{(1)}\left(\psi, A_{C}^{\mu, a}\right)=0(\text { static }) \\
& \mathscr{L}_{Q-C}^{(1)}\left(\psi, A_{C}^{\mu, a}\right)=g \sum_{\mathrm{p}, \mathrm{q}_{T}} \psi_{\mathrm{p}+\mathrm{q}_{T}}^{\dagger}\left(\frac{2 \mathrm{~A}_{C} \cdot \mathscr{P}+\left[\mathscr{P} \cdot \mathrm{A}_{C}\right]-i\left[\mathscr{P} \times \mathrm{A}_{C}\right] \cdot \sigma}{2 m}\right) \psi_{\mathrm{p}}(\text { soft }),
\end{aligned}
$$

where we use squared brackets in order to denote the region in which the label momentum operator, $\mathscr{P}^{\mu}$, acts. If we consider the non-relativistic limit of the $t$-channel gluon exchange diagram for a particular source, in addition to the above rules we obtain explicit expressions for the Glauber and Coulomb fields $A_{G}^{\mu, a}, A_{C}^{\mu, a}$. The interested reader can find those results in [10].

\section{Conclusions}

Theory, phenomenology, and experimental measurements of quarkonia have gone a long way since the early investigation of $J / \psi$ production in fixed target experiments at CERN and the works by Gavin, Gyulassy and Jackson. In these proceedings I reported on the derivation of the leading and sub-leading Lagrangians of $\mathrm{NRQCD}_{\mathrm{G}}$ for a single virtual gluon exchange [10]. This was achieved using three different approaches: i) the background field method, ii) a matching (with QCD) procedure, and iii) a hybrid method. Explicit results for the Glauber and Coulomb fields were also obtained. Even though I described the formal aspects of of $\mathrm{NRQCD}_{\mathrm{G}}$, it can be easily seen that the phenomenological studies of ground and excite $J / \psi$ and $\Upsilon$ states that we have done in the past $[24,25]$ correspond to the leading medium correction $\mathscr{L}_{Q-G / C}^{(0)}\left(\psi, A_{G / C}^{\mu, a}\right)$ and diagonal quarkonim state to quarkonium state transitions. With the new theoretical framework at hand, such calculations can be extended rigorously to different systems and to include medium-induced transitions from and to exited states.

\section{References}

[1] X.-N. Wang, 30 years of jet quenching, in 13th International Workshop on High-pT Physics in the RHIC/LHC Era (HPT 2019) Knoxville, TN, USA, March 19-22, 2019, 2019, 1906.11998.

[2] S. Gavin and M. Gyulassy, Transverse Momentum Dependence of J/psi Production in Nuclear Collisions, Phys. Lett. B214 (1988) 241.

[3] S. Gavin, M. Gyulassy and A. Jackson, Hadronic J/psi Suppression in Ultrarelativistic Nuclear Collisions, Phys. Lett. B207 (1988) 257.

[4] CMS collaboration, Event activity dependence of $Y(n S)$ production in $\sqrt{s_{N N}}=5.02 \mathrm{TeV} \mathrm{pPb}$ and $\sqrt{s}=2.76 \mathrm{TeV}$ pp collisions, JHEP 04 (2014) 103 [1312.6300]. 
[5] PHENIX collaboration, Nuclear Modification of ??,? $?_{c}$, and J/? Production in $d+A u$ Collisions at $\sqrt{s_{N N}}=200$ ??GeV, Phys. Rev. Lett. 111 (2013) 202301 [1305.5516].

[6] STAR collaboration, Suppression of $\Upsilon$ production in $d+A u$ and $A u+A u$ collisions at $\sqrt{s_{N N}}=200 \mathrm{GeV}$, Phys. Lett. B735 (2014) 127 [1312.3675].

[7] G. Ovanesyan and I. Vitev, An effective theory for jet propagation in dense QCD matter: jet broadening and medium-induced bremsstrahlung, JHEP 06 (2011) 080 [1103 . 1074].

[8] Z.-B. Kang, F. Ringer and I. Vitev, Effective field theory approach to open heavy flavor production in heavy-ion collisions, JHEP 03 (2017) 146 [1610. 02043$].$

[9] G. T. Bodwin, E. Braaten and G. P. Lepage, Rigorous QCD analysis of inclusive annihilation and production of heavy quarkonium, Phys. Rev. D51 (1995) 1125 [hep-ph/9407339].

[10] Y. Makris and I. Vitev, An Effective Theory of Quarkonia in QCD Matter, 1906.04186.

[11] X.-N. Wang and M. Gyulassy, Gluon shadowing and jet quenching in A $A$ collisions at $s^{* *}(1 / 2)=$ 200-GeV, Phys. Rev. Lett. 68 (1992) 1480.

[12] M. Gyulassy, I. Vitev, X.-N. Wang and B.-W. Zhang, Jet quenching and radiative energy loss in dense nuclear matter, nucl-th/0302077.

[13] M. Spousta, On similarity of jet quenching and charmonia suppression, Phys. Lett. B767 (2017) 10 [1606.00903].

[14] F. Arleo, Quenching of Hadron Spectra in Heavy Ion Collisions at the LHC, Phys. Rev. Lett. 119 (2017) 062302 [1703.10852].

[15] CMS collaboration, Suppression and azimuthal anisotropy of prompt and nonprompt $\mathrm{J} / \psi$ production in PbPb collisions at $\sqrt{s_{N N}}=2.76 \mathrm{TeV}$, Eur. Phys. J. C77 (2017) 252 [1610.00613].

[16] ATLAS collaboration, Prompt and non-prompt $J / \psi$ and $\psi(2 \mathrm{~S})$ suppression at high transverse momentum in $5.02 \mathrm{TeV} \mathrm{Pb+Pb}$ collisions with the ATLAS experiment, Eur. Phys. J. C78 (2018) 762 [1805.04077].

[17] G. C. Nayak, J.-W. Qiu and G. F. Sterman, Fragmentation, factorization and infrared poles in heavy quarkonium production, Phys. Lett. B613 (2005) 45 [hep-ph/ 0501235 ].

[18] S. Fleming, A. K. Leibovich, T. Mehen and I. Z. Rothstein, The Systematics of Quarkonium Production at the LHC and Double Parton Fragmentation, Phys. Rev. D86 (2012) 094012 [1207.2578].

[19] G. T. Bodwin, K.-T. Chao, H. S. Chung, U.-R. Kim, J. Lee and Y.-Q. Ma, Fragmentation contributions to hadroproduction of promptJ $/ \psi, \chi_{c J}$, and $\psi(2 S)$ states, Phys. Rev. D93 (2016) $034041[1509.07904]$.

[20] H. T. Li and I. Vitev, Inverting the mass hierarchy of jet quenching effects with prompt b-jet substructure, Phys. Lett. B793 (2019) 259 [1801. 00008 ].

[21] Z.-B. Kang, J. Reiten, I. Vitev and B. Yoon, Light and heavy flavor dijet production and dijet mass modification in heavy ion collisions, Phys. Rev. D99 (2019) 034006 [1810 . 10007 ].

[22] H. T. Li and I. Vitev, Inclusive heavy flavor jet production with semi-inclusive jet functions: from proton to heavy-ion collisions, 1811.07905.

[23] I. Z. Rothstein, P. Shrivastava and I. W. Stewart, Manifestly Soft Gauge Invariant Formulation of vNRQCD, 1806.07398. 
[24] R. Sharma and I. Vitev, High transverse momentum quarkonium production and dissociation in heavy ion collisions, Phys. Rev. C87 (2013) 044905 [1203.0329].

[25] S. Aronson, E. Borras, B. Odegard, R. Sharma and I. Vitev, Collisional and thermal dissociation of $J / \psi$ and $\Upsilon$ states at the LHC, Phys. Lett. B778 (2018) 384 [1709. 02372]. 